01

\title{
Внутридопплеровские резонансы, обусловленные осцилляциями Раби на атомных переходах в тонких газовых ячейках
}

\author{
(C) А.Ч. Измайлов \\ Институт фризики НАН Азербайджана, \\ AZ-1143 Баку, Азербайджан \\ e-mail: azizm57@rambler.ru
}

Поступила в редакцию 08.01.2019 г.

В окончательной редакции 08.01.2019 г.

Принята к публикации 23.01.2019 г.

\begin{abstract}
Теоретически исследовано возбуждение атомов из основного квантового уровня в метастабильное состояние бегущим монохроматическим излучением в тонкой газовой ячейке, внутренняя толщина которой во много раз меньше ее радиуса. Обнаружены и проанализированы внутридопплеровские резонансы, возникающие в населенности метастабильного атомного уровня при сканировании частоты излучения вследствие светоиндуцированных осцилляций Раби на данном оптическом переходе, а также специфики пролетной релаксации атомов в рассматриваемой ячейке с разреженным газом. Такие нетривиальные резонансы могут быть зарегистрированы во флуоресценции атомов с помощью дополнительного пробного светового пучка. Предложен метод непосредственного измерения частоты Раби по спектральным интервалам между установленными внутридопплеровскими резонансами. Полученные результаты могут найти применение в атомной спектроскопии для прецизионного определения компонент матричных элементов дипольных моментов, а также связанных с ними коэффициентов Эйнштейна и сил осцилляторов квантовых переходов между основным и метастабильными уровнями атомов.
\end{abstract}

DOI: 10.21883/OS.2019.06.47761.5-19

\section{Введение}

Обычно спектроскопические исследования атомов и молекул проводятся в газовых ячейках, длина $L$ которых гораздо больше их радиуса $R[1]$. Вместе с тем новые возможности лазерной спектроскопии высокого разрешения открываются при использовании тонких ячеек с разреженной газовой средой, для размеров которых выполняется обратное соотношение $L \ll R$. Так, в обзорах [2,3] описаны результаты теоретических и экспериментальных исследований нетривиальных внутридопплеровских резонансов поглощения, дисперсии и флуоресценции, которые возникают на центральных частотах квантовых переходов атомов (молекул) вследствие особенностей их оптической накачки и пролетной релаксации в таких тонких ячейках. В частности, представляет интерес анализ узких спектральных структур, обусловленных спецификой селекции атомов по скоростям на оптически возбужденных квантовых уровнях, у которых время радиационного распада велико по сравнению с характерным временем пролета атомов между стенками ячейки с линейными размерами в пределах нескольких сантиметров. Такая ситуация может иметь место для многих атомов и молекул, если лазерное излучение резонансно переходу, который связывает какой-либо подуровень $a$ основного квантового терма с достаточно долгоживущим (метастабильным) уровнем $b$ [4]. Соответствующие весьма узкие резонансы на таких „запрещенных“ переходах в тонких газовых ячейках начали теоретически исследоваться в работе [5]. При этом рассматривалась разреженная газовая среда в цилиндрической ячейке с радиусом $R$ и внутренней толщиной $L \ll R$ (рис. 1). Данная ячейка облучается вдоль оси $z$ бегущим монохроматическим лазерным пучком, частота $\omega$ которого сканировалась в окрестности центра $\omega_{0}$ перехода $a \rightarrow b$ из основного атомного состояния $a$ на метастабильный уровень $b$. Отмеченная работа [5] была нацелена на поиск и анализ новых атомных спектральных реперов для высокоточных стандартов частоты. Таким образом, в данной работе [5] проводились исследования наиболее узких внутридопплеровских резонансов, которые индуцируются лазерным пучком накачки, пространственно отделенным от центральной области регистрации в данной ячейке. Вследствие этого в исследованных резонансах был пренебрежимо мал вклад атомов в оптически возбужденном метастабильном состоянии $b$, пролетающих между плоскопараллельными стенками ячейки (рис. 1) с характерным временем $L /\left|v_{z}\right|$, где $v_{z}$ - проекция атомной скорости на волновой вектор $\mathbf{k}$ пучка накачки. Заметим однако, что при однородном лазерном облучении всего объема рассматриваемой ячейки за время $L /\left|v_{z}\right|$ между населенностями уровней $a$ и $b$ резонансного перехода $a \rightarrow b$ происходят светоиндуцированные осцилляции Раби [1], количество которых определяется величиной $g L /\left|v_{z}\right|$, где $g$ - частота Раби. Вместе с тем в случае допплеровски уширенной спектральной линии перехода $a \rightarrow b$ монохроматическое излучение накачки эффективно взаимодействует лишь с атомами, проекции скорости которых $v_{z}$ близки к 


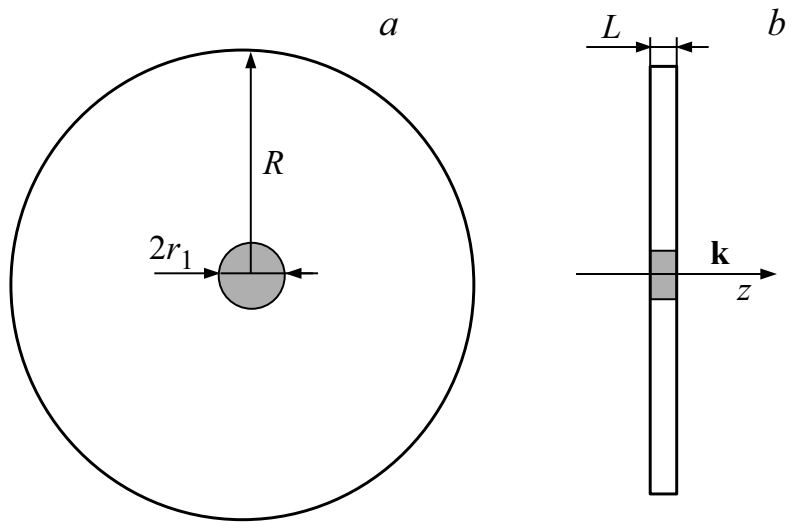

Рис. 1. Схема тонкой газовой ячейки с внутренней толщиной $L$ и радиусом $R \gg L$ в двух ортогональных проекциях $(a)$ и $(b)$. Данная ячейка облучается по всему объему бегущим вдоль оси $z$ монохроматическим лазерным излучением с волновым вектором $\mathbf{k}$. Регистрирующий пробный световой пучок с радиусом $r_{1}$ проходит через центральную область ячейки.

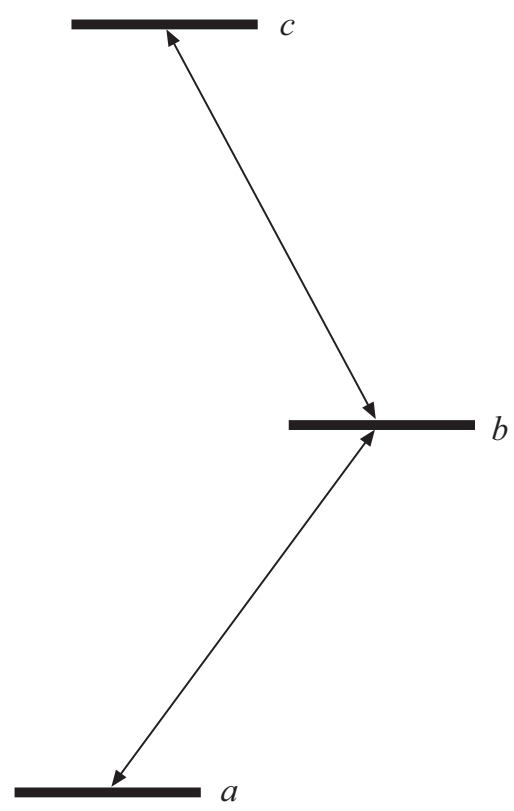

Рис. 2. Схема рассматриваемых квантовых уровней и переходов: $a \rightarrow b$-переход из подуровня $a$ основного терма в метастабильное состояние $b$, который осуществляется монохроматическим излучением накачки; $b \rightarrow c$-переход, индуцируемый пробным световым пучком для регистрации сигнала флуоресценции из возбужденного уровня $c$.

значению $\left(\omega-\omega_{0}\right) / k$ [1]. Поэтому при сканировании расстройки частоты $\delta=\left(\omega-\omega_{0}\right)$ возможно появление новых внутридопплеровских резонансов в населенности метастабильного уровня $b$, существенно зависящих от фактора $g L /\left|v_{z}\right| \sim g k L|\delta|^{-1}$.

Однако регистрация подобных резонансов непосредственно по спектру поглощения или рассеяния излучения накачки не будет эффективной вследствие слабости „запрещенного“ перехода $a \rightarrow b$. Согласно мето- ду, предложенному в работе [5], для таких измерений целесообразно использовать дополнительный пробный световой пучок, проходящий через центральную область газовой ячейки с радиусом $r_{1}$ (рис. 1). Такое пробное излучение индуцирует переход $b \rightarrow c$ из метастабильного состояния $b$ на какой-либо другой возбужденный уровень $c$ с достаточно малым временем радиационного распада (рис. 2), из которого и будет регистрироваться соответствующий спектр флуоресценции.

В связи с вышесказанным в настоящей работе проведено теоретическое исследование новых внутридопплеровских резонансов, обусловленных осцилляциями Раби в атомах разреженной газовой среды в тонкой газовой ячейке (рис. 1). Показана возможность непосредственного измерения частоты Раби $g$ для ,запрещенного“ атомного перехода $a \rightarrow b$ по частотным интервалам между данными нетривиальными резонансами.

\section{Основные соотношения}

Рассмотрим облучение разреженного газа монохроматическим лазерным пучком накачки в тонкой прозрачной цилиндрической ячейке с радиусом $R$ и внутренней толщиной $L \ll R$ (рис. 1). Этот пучок распространяется вдоль оси $z$ в ортогональном направлении к плоскопараллельным стенкам ячейки. Считается, что интенсивность данного пучка одинакова во всем объеме ячейки. Тогда электрическая компонента рассматриваемого монохроматического излучения накачки описывается следующей формулой в цилиндрических координатах $r$ и $z$ :

$$
\mathbf{Q}(z, r, t)=0.5 \mathbf{E} \eta(R-r) \exp [i(\omega t-k z)]+c . c .,
$$

где $\mathbf{E}=E \mathbf{e}, E$ и $\mathbf{e}-$ соответственно амплитуда и единичный вектор поляризации, $\omega$ - его частота, $k=\frac{2 \pi}{\lambda}-$ волновое число, $\lambda$ - длина волны, а $\eta(x)-$ ступенчатая функция $(\eta(x)=1$, если $x \geq 0$ и $\eta(x)=0$, когда $x<0)$.

Частота пучка $\omega$ близка к центру $\omega_{0}$ перехода $a \rightarrow b$ между подуровнем $a$ основного квантового терма и возбужденным метастабильным состоянием $b$ атомов. Плотность газа в ячейке полагается настолько низкой, что можно пренебречь межатомными столкновениями. Также не будем принимать во внимание эффекты светового давления на атомы. Рассмотрим ситуацию, когда время жизни возбужденного уровня $b$ гораздо больше, чем характерное время $2 R / u$ пролета атомов через ячейку (рис. 1) с наиболее вероятной скоростью $u$. Тогда можно не учитывать радиационную релаксацию этого уровня $b$.

При данных условиях взаимодействие светового пучка (1) с атомами в тонкой газовой ячейке (рис. 1) может быть описано следующей системой уравнений для элементов матрицы плотности оптической когерентности 
$\rho_{a b}$, а также населенностей $\rho_{a}$ и $\rho_{b}$ уровней $a$ и $b[6]$ :

$$
\begin{gathered}
\rho_{a}+\rho_{b}=N F(\mathbf{v}), \\
\frac{\partial \rho_{b}}{\partial t}+\mathbf{v} \frac{\partial \rho_{b}}{\partial \mathbf{r}}=-0.5 i g \rho_{a b}^{*} \exp [i(\delta t-k z)]+c . c ., \\
\frac{\partial \rho_{a b}}{\partial t}+\mathbf{v} \frac{\partial \rho_{a b}}{\partial \mathbf{r}}=0.5 i g\left(\rho_{b}-\rho_{a}\right) \exp [i(\delta t-k z)]+c . c .
\end{gathered}
$$

где $N$ - плотность атомов в основном состоянии $a$ при отсутствии лазерного излучения (1), $F(\mathbf{v})$ - распределение Максвелла по атомной скорости $\mathbf{v}, g=\frac{(\mathbf{E d})}{\hbar}-$ частота Раби, d - матричный элемент дипольного момента для перехода $a \rightarrow b, \delta=\left(\omega-\omega_{0}\right)-$ расстройка частоты. Уравнения (2) должны быть дополнено граничными условиями, которые зависят от особенностей столкновений атомов со стенками ячейки. Как и в предыдущих теоретических исследованиях $[2,3,5]$ для тонких газовых ячеек, будем считать, что в результате таких столкновений устанавливается равновесное распределение как по населенностям квантовых уровней, так и по скоростям атомов. Таким образом, полагается, что элементы матрицы плотности $\rho_{b}$ и $\rho_{a b}$ обращаются в нуль на стенках ячейки. При данных условиях мы получаем из уравнений (2) следующее выражение для населенности $\rho_{b}^{(0)}$ возбужденных атомов на центральной оси $z$ лазерного пучка в интервале $0 \leq z \leq L$ (рис. 1):

$$
\begin{aligned}
\rho_{b}^{(0)}= & N F_{l}\left(v_{z}\right) F_{t}\left(v_{t}\right) g^{2} \Omega^{-2}\left[\beta\left(z, v_{z}, v_{t}\right) \eta\left(v_{z}\right)\right. \\
& \left.+\beta\left(L-z,-v_{z}, v_{t}\right) \eta\left(-v_{z}\right)\right],
\end{aligned}
$$

где

$$
\begin{aligned}
\beta\left(z, v_{z}, v_{t}\right)= & \sin ^{2}\left(\frac{\Omega z}{2 v_{z}}\right) \eta\left(\frac{R}{v_{t}}-\frac{z}{v_{z}}\right) \\
& +\sin ^{2}\left(\frac{\Omega R}{2 v_{t}}\right) \eta\left(\frac{z}{v_{z}}-\frac{R}{v_{t}}\right)
\end{aligned}
$$

$\Omega=\left[g^{2}+\left(\delta-k v_{z}\right)^{2}\right]^{0.5}$, величины $v_{z}$ и $v_{t}$ являются соответственно продольной и поперечной (радиальной) компонентами атомной скорости $\mathbf{v}$, которые характеризуются известными максвелловскими распределениями $F_{l}\left(v_{z}\right)$ и $F_{t}\left(v_{t}\right)$

$$
\begin{gathered}
F_{l}\left(v_{z}\right)=\pi^{-0.5} u^{-1} \exp \left(-v_{z}^{2} u^{-2}\right) \\
F_{t}\left(v_{t}\right)=2 v_{t} u^{-2} \exp \left(-v_{t}^{2} u^{-2}\right)
\end{gathered}
$$

Далее получаем среднее значение $\left\langle\rho_{b}^{(0)}\right\rangle$ населенности $\rho_{b}^{(0)}(3)$ по длине ячейки $L$ :

$$
\begin{aligned}
& \left\langle\rho_{b}^{(0)}\right\rangle=L^{-1} \int_{0}^{L} \rho_{b}^{(0)}(z) d z=0.5 N F_{l}\left(v_{z}\right) F_{t}\left(v_{t}\right) g^{2} \Omega^{-2} \\
& \times\left\{\left[1-\frac{\left|v_{z}\right|}{\Omega L} \sin \left(\frac{\Omega L}{\left|v_{z}\right|}\right)\right] \eta\left(\frac{R\left|v_{z}\right|}{L v_{t}}-1\right)\right. \\
& +\left[1-\frac{\left|v_{z}\right|}{\Omega L} \sin \left(\frac{\Omega R}{v_{t}}\right)-\left(1-\frac{R\left|v_{z}\right|}{L v_{t}}\right) \cos \left(\frac{\Omega R}{v_{t}}\right)\right] \\
& \left.\quad \times \eta\left(1-\frac{R\left|v_{z}\right|}{L v_{t}}\right)\right\} .
\end{aligned}
$$

Заметим, что атомы, пролетающие между плоскопараллельными стенками ячейки (рис. 1), определяют величину $\left\langle\rho_{b}^{(0)}\right\rangle(6)$ при условии $\left|v_{z}\right|>(L / R) v_{t}$ для размеров этой ячейки и компонент атомной скорости $v_{z}$ и $v_{t}$. Обратное соотношение $\left|v_{z}\right|<(L / R) v_{t}$ в (6) соответствует атомам, траектории полета которых начинаются или заканчиваются на цилиндрической поверхности рассматриваемой ячейки (рис. 1).

Средняя населенность $N_{b}$ всевозможных атомов в метастабильном состоянии $b$ на центральной оси ячейки получается после интегрирования величины $\left\langle\rho_{b}^{(0)}\right\rangle(6)$ по проекциям атомных скоростей:

$$
N_{b}=\int_{-\infty}^{\infty}\left[\int_{0}^{\infty}\left\langle\rho_{b}^{(0)}\right\rangle d v_{t}\right] d v_{z}
$$

Следует отметить, что соотношения (3)-(7) непосредственно обобщаются также на случай магнитодипольного перехода $a \rightarrow b$ между основным и метастабильным квантовыми состояниями $a$ и $b$. Тогда соответствующая частота Раби $g=(\mathbf{H} \boldsymbol{\mu}) / \hbar$ определяется магнитной составляющей Н излучения накачки и матричным элементом $\boldsymbol{\mu}$ магнитного дипольного момента данного перехода.

Поскольку выражения (6), (7) для искомой населенности метастабильных атомов определяются модулем частоты Раби, то далее будем считать величину $g$ положительной.

\section{Обсуждение результатов}

В соответствии с методом, предложенным в работе [5], считается, что через центральную область ячейки направляется пробный световой пучок сравнительно малого радиуса $r_{1} \ll R$ (рис. 1$)$, который индуцирует оптический переход $b \rightarrow c$ из метастабильного состояния $b$ на другой возбужденный атомный уровень $c$ (рис. 2) с достаточно коротким временем радиационного распада. Интенсивность данного пробного излучения полагается 

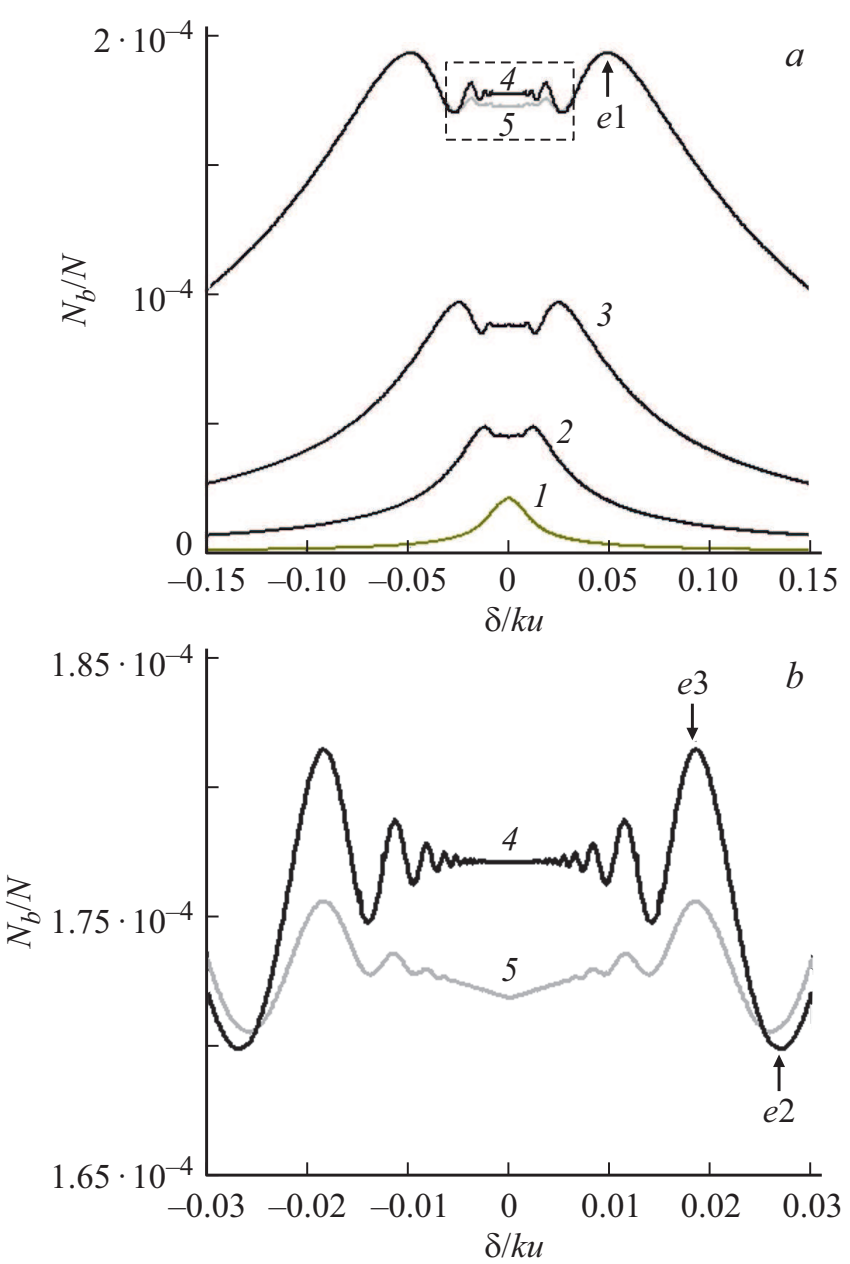

Рис. 3. (a) Зависимость населенности $N_{b}(\delta)$ метастабильного уровня $b$ (в единицах атомной плотности $N$ ) от расстройки частоты $\delta$ (в единицах допплеровского уширения $k u$ ), когда $L=150 \lambda, \quad R=100 L \quad(1-4), 40 L \quad(5), \quad(g / k u)=2 \cdot 10^{-5} \quad(1)$, $5 \cdot 10^{-5}(2), 10^{-4}(3)$ и $2 \cdot 10^{-4}(4,5)$. (b) Фрагмент рис. $3, a$, обозначенный прямоугольником, в увеличенном масштабе. Стрелками показано положение трех наиболее заметных внутридопплеровских резонансов $(e 1, e 2, e 3)$, обусловленных осцилляциями Раби.

настолько низкой, что несущественно изменение заселенности метастабильного уровня $b$ в процессе пролета атомов через область регистрации в центре ячейки (рис. 1). Тогда при сканировании расстройки частоты $\delta=\left(\omega-\omega_{0}\right)$ спектральный профиль регистрируемого сигнала флуоресценции из уровня $c$ (рис. 2) будет подобен зависимости населенности $N_{b}(\delta)(7)$ метастабильных атомов от $\delta$.

Рисунок 3 представляет такие численно рассчитанные частотные зависимости $N_{b}(\delta)(7)$ от расстройки частоты $\delta$ при различных интенсивностях излучения накачки и отношений $L / R$ внутренней толщины ячейки $L$ к ее радиусу $R$ (рис. 1$)$. Мы рассматриваем случай, когда допплеровское уширение $k u$ спектральной линии перехода $a \rightarrow b$ во много раз превосходит частоту Раби $g$. Тогда может возникнуть серия внутридопплеровских резонан- сов, симметрично расположенных относительно нулевой расстройки $\delta=0$ (рис. 3). Видно, что положение, амплитуда, ширина, а также количество этих нетривиальных резонансов существенно зависят от значения частоты Раби $g$. Действительно, за время пролета $L /\left|v_{z}\right|$ атомов между плоскопараллельными стенками рассматриваемой ячейки (рис. 1) происходят светоиндуцированные осцилляции населенностей между уровнями $a$ и $b$ перехода $a \rightarrow b[1,6]$. Число таких осцилляций определяется величиной $g L /\left|v_{z}\right|$. Для рассматриваемой допплеровски уширенной спектральной линии монохроматическое излучение накачки эффективно взаимодействует только с атомами, проекции скоростей $v_{z}$ которых находятся в узкой окрестности значения $\delta / k[1,6]$. Поэтому за время пролета $L /\left|v_{z}\right|$ атомов между плоскопараллельными стенками газовой ячейки (рис. 1) с резонансной проекцией скорости $v_{z}=\delta / k$ происходит целое число $|n|$ осцилляций Раби между нулевым и максимальным значениями населенности метастабильного состояния $b$, если $g L /\left|v_{z}\right|=\pi|n|$, т.е. при следующих расстройках частоты:

$$
\delta_{n}=\frac{g k L}{\pi n}=\frac{2 g}{n}\left(\frac{L}{\lambda}\right) \quad(n= \pm 1, \pm 2 \ldots) .
$$

Согласно соотношению (6), анализируемая населенность $N_{b}(7)$ определяется метастабильными атомами, пролетающими непосредственно между плоскопараллельными стенками ячейки (рис. 1), только при условии $\left|v_{z}\right|>(L / R) v_{t}$ для компонент атомной скорости. Данное условие выполняется при расстройках частоты $|\delta|>(L / R) k u$. Поэтому мы не наблюдаем проявлений осцилляций Раби при достаточно низкой интенсивности излучения накачки, когда максимальное из значений расстроек $\left|\delta_{n}\right|(8)$ с $n= \pm 1$ оказывается меньше, чем $(L / R) k u$, т.е. если $(g / k u)<0.5(\lambda / R)$ (кривая 1 на рис. 3,a). Внутридопплеровские резонансы, обусловленные осцилляциями Раби, начинают последовательно возникать с повышением интенсивности накачки по мере выполнения для расстроек (8) соотношений $\left|\delta_{n}\right| \geq(L / R) k u$, т. е. когда $(g / k u)>0.5|n|(\lambda / R)$ при $|n|=1,2,3, \ldots$ Тогда увеличивается не только количество данных резонансов, но также возрастает их амплитуда и ширина, причем эти резонансы сдвигаются к более высоким значениям расстройки частоты (рис. $3, a$ ). Из сравнения зависимостей 4 и 5 на рис. $3, a$ и $b$, полученных для тонких ячеек (рис. 1) с одинаковой внутренней толщиной $L$, но разными радиусами $R$, видно, что внутридопплеровские резонансы, обусловленные осцилляциями Раби, лучше проявляются при меньшем отношении $(L / R) \ll 1$. При сравнительно малых частотных расстройках $|\delta|<(L / R) k u$ в спектральный контур $N_{b}(\delta)(7)$ основной вклад дают метастабильные атомы, траектории полета которых начинаются или заканчиваются на цилиндрической поверхности ячейки (рис. 1). Поэтому резонансы, обусловленные осцилляциями Раби, практически не проявляются в спектральном интервале 


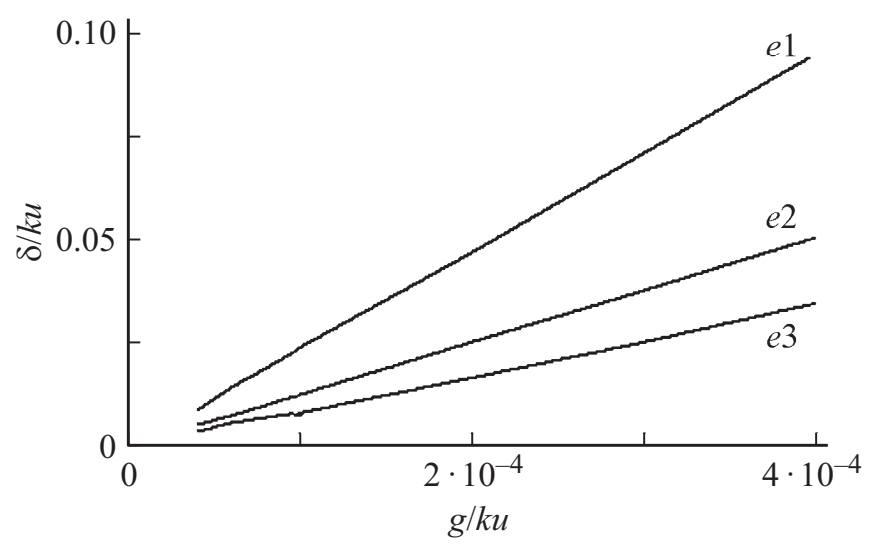

Рис. 4. Зависимость (в единицах допплеровского уширения $k u$ ) положения экстремумов $\delta e 1, \delta e 2$ и $\delta e 3$ трех внутридопплеровских резонансов $(e 1, e 2, e 3)$, отмеченных стрелками на рис. 3 , от частоты Раби $g ; L=150 \lambda, R=100 L$.

$|\delta|<(L / R) k u$ при любой интенсивности излучения накачки (рис.3). С ростом расстройки частоты $\delta$ в области $|\delta|>\left|\delta_{ \pm 1}\right|=2 g(L / \lambda)(8)$ происходит монотонное убывание величины $N_{b}(\delta)$ (рис. $\left.3, a\right)$ вследствие уменьшения времени $L /\left|v_{z}\right|$ эффективного оптического возбуждения на рассматриваемом переходе $a \rightarrow b$ в процессе пролета атомов между стенками ячейки с проекциями скорости $v_{z}$, близкими к резонансному значению $\delta / k$.

По частотным интервалам между экстремумами внутридопплеровских резонансов в спектральных зависимостях $N_{b}(\delta)$ (рис. 3) можно непосредственно определить значение частоты Раби $g$. Так, на рис. 4 представлены зависимости от $g$ положения экстремумов $\delta e 1, \delta e 2, \delta e 3$ трех наиболее заметных резонансов, отмеченных стрелками на рис. 3. Данные зависимости являются квазилинейными в области четкого проявления указанных резонансов. Например, при значении $(g / k u) \geq 3 \cdot 10^{-4}$ на рис. 4 они достаточно хорошо описываются соотношениями $|\delta e 1| \approx 1.59 \mathrm{~g}(L / \lambda)$, $|\delta e 2| \approx 0.86 g(L / \lambda)$ и $|\delta e 3| \approx 0.61 g(L / \lambda)$. Заметим, что каждая из этих величин $\delta e n(\mathrm{c} n=1,2,3)$ находится в интервале между соответствующими значениями расстроек $\delta n$ и $\delta(n+1)(8)$. Посредством незначительной модуляции расстройки частоты $\delta$ на эксперименте можно получить зависимость от $\delta$ непосредственно производной $\frac{d N_{b}(\delta)}{d \delta}$, где гораздо лучше проявятся более узкие, но менее заметные внутридопплеровские резонансы из спектра $N_{b}(\delta)$ на рис. 3. Таким образом, возникает целая серия частотных реперов для высокоточного измерения частоты Раби $g$ при известных параметрах излучения накачки и размеров тонкой газовой ячейки (рис. 1). В частности, для подобных измерений могут быть использованы спектральные интервалы между какой-либо парой внутридопплеровских резонансов, симметрично расположенных относительно нулевой расстройки $\delta=0$ (рис. 3). Прежде всего это относится к интервалу $2 \delta e 1$ между экстремумами двух таких наиболее круп- ных резонансов (рис. 3,a). Следует, однако, отметить, что оценки возможных погрешностей таких измерений на эксперименте потребуют дополнительного анализа. В частности, определение частоты Раби $g$ предложенным способом будет эффективным только при условии поддержания практически одинаковой интенсивности $I$ излучения накачки по всему сечению тонкой ячейки (рис. 1 ), поскольку $g \sim I^{0.5}$.

Представленные на рис. 3 и 4 результаты численных расчетов характерны, например, для тонкой ячейки (рис. 1) с толщиной $L=150 \lambda \approx 0.1 \mathrm{~mm}$ и радиусом $R=100 L \approx 1 \mathrm{~cm}$, содержащей разреженные пары кальция, так как у изотопа ${ }^{40} \mathrm{Ca}$ есть соответствующий интеркомбинационный переход ${ }^{1} S_{0} \rightarrow{ }^{3} P_{1}$ (с длиной волны $\lambda=657 \mathrm{~nm})$ из основного состояния ${ }^{1} S_{0}$ на метастабильный уровень ${ }^{3} P_{1}$ [4]. Заметим, что линейно или циркулярно поляризованное излучение накачки индуцирует переход только между двумя определенными зеемановскими подуровнями данных уровней ${ }^{1} S_{0}$ и ${ }^{3} P_{1}$, имеющих полные угловые моменты соответственно 0 и 1 . Это также согласуется с рассмотренной в настоящей работе моделью невырожденных уровней $a$ и $b$ резонансного перехода $a \rightarrow b$. Вместе с тем полученные здесь результаты могут быть обобщены и на другие подобные „запрещенные“ оптические переходы, которые имеют место, в частности, в атомах магния, стронция, бария и иттербия [4]. При наличии зеемановского вырождения уровней $a$ и $b$ можно посредством внешнего постоянного магнитного поля $\mathbf{H}_{0}$ расщепить спектральную линию перехода $a \rightarrow b$ на отдельные компоненты $a \mu \rightarrow b m$, связывающие подуровни $a \mu$ и $b m$ с определенными проекциями $\mu$ и $m$ углового момента на вектор $\mathbf{H}_{0}$. После этого становится возможным измерение частоты Раби $g_{\mu m}$ для выделенного перехода $a \mu \rightarrow b m$ рассмотренным выше способом.

Как отмечалось ранее, внутридопплеровские резонансы в спектральной зависимости $N_{b}(\delta)$ (рис. 3) можно зарегистрировать высокочувствительным методом флуоресценции из возбужденного уровня $c$, который заселяется пробным излучением на переходе $b \rightarrow c$ из метастабильного уровня $b$ (рис. 2). Например, в случае упомянутого метастабильного уровня ${ }^{3} P_{1}$ атомов кальция для этого могут быть использованы следующие достаточно сильные оптические переходы: ${ }^{3} P_{1} \rightarrow{ }^{3} S_{1}$ (с длиной волны $612.2 \mathrm{~nm}),{ }^{3} P_{1} \rightarrow{ }^{3} D_{2}(443.5 \mathrm{~nm})$ или ${ }^{3} P_{1} \rightarrow{ }^{3} P_{0}(430 \mathrm{~nm})[4]$.

При известной интенсивности и поляризации излучения накачки по измеренному на эксперименте рассмотренным методом абсолютному значению частоты Раби $g$ можно непосредственно найти соответствующий модуль векторной компоненты матричного элемента дипольного момента „запрещенного“ перехода $a \rightarrow b$, а следовательно, определить коэффициенты Эйнштейна и силу осциллятора данного перехода. 


\section{Список литературы}

[1] Demtroder W. Laser Spectroscopy: Basic Concepts and Instrumentation. Springer-Verlag, 1996. 924 p.

[2] Izmailov A.Ch. // Proc. SPIE. 2007. V. 6727. P. 67270B.

[3] Izmailov A.Ch. // Azerbaijan J. of Phys.: FIZIKA. 2011. V. 17. N 1. Sec. EN. P. 3.

(режим доступа: physics.gov.az/index1.html).

[4] Radzig A.A., Smirnov B.M. Reference data on atoms, molecules and ions. Berlin, Heidelberg: Springer-Verlag, 1985. 465 p.

[5] Beverini N., Izmailov A.Ch. // Opt. Commun. 2009. V. 282. P. 2527.

[6] Летохов В.С., Чеботаев В.П. Нелинейная лазерная спектроскопия сверхвысокого разрешения. М.: Наука, 1990. 512 c. Letokhov V.S., Chebotayev V.P. Nonlinear Laser Spectroscopy. Berlin: Springer-Verlag, 1977. 466 p. 